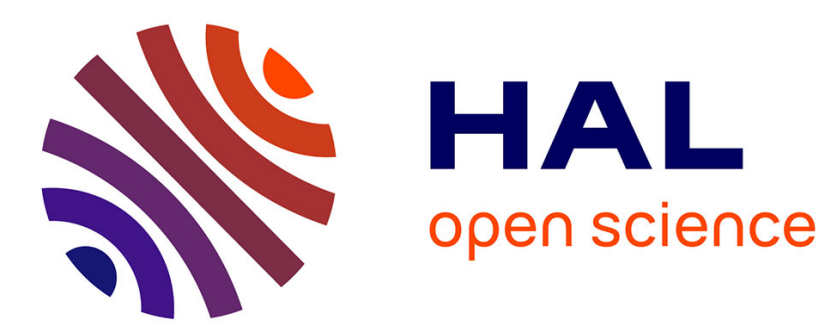

\title{
ON AN ARITHMETICAL QUESTION RELATED TO PERFECT NUMBERS
}

\author{
Paul Lescot
}

\section{To cite this version:}

Paul Lescot. ON AN ARITHMETICAL QUESTION RELATED TO PERFECT NUMBERS. Mathematical Gazette, In press. hal-02050720

\section{HAL Id: hal-02050720 \\ https://hal.science/hal-02050720}

Submitted on 27 Feb 2019

HAL is a multi-disciplinary open access archive for the deposit and dissemination of scientific research documents, whether they are published or not. The documents may come from teaching and research institutions in France or abroad, or from public or private research centers.
L'archive ouverte pluridisciplinaire HAL, est destinée au dépôt et à la diffusion de documents scientifiques de niveau recherche, publiés ou non, émanant des établissements d'enseignement et de recherche français ou étrangers, des laboratoires publics ou privés. 


\section{ON AN ARITHMETICAL QUESTION RELATED TO PERFECT NUMBERS}

\section{IntRoduction AND STATEMENT OF THE PROBLEM}

For $n \geq 1$ an integer, we shall denote by $\sigma(n)$ the sum of the (positive) divisors of $n$.

The line of thought leading to this note started with [3, Exemple III, p. 380], where two integers $k$ such that $\sigma\left(k^{3}\right)$ is a perfect square are given.

This suggests that we might look for numbers $n$ such that $\sigma(n)$ be a square. One rapidly notices that $\sigma(66)=144=12^{2}$; furthermore

$$
\sigma(66)-2.66=144-132=12,
$$

whence

$$
\sigma(66)=(\sigma(66)-2.66)^{2}
$$

We were thus led to the following definition :

Definition. An integer $n$ is termed quadratically perfect if

$$
\sigma(n)=(\sigma(n)-2 n)^{2}
$$

As we have just established, 66 is such a number. A quick search yields three others : 1,3 and 491536.

As a matter of fact, one has

Lemma 1. The only quadratically perfect primary integers are 1 and 3 .

Proof. Let $n=p^{k}$ ( $p$ prime) be quadratically perfect ; if $k=0, n=1$. Let us then assume $k \geq 1$; we have

whence, setting $S:=\sum_{l=0}^{k-1} p^{l}$, we find

$$
\sigma(n)=1+p+\ldots+p^{k}
$$

$$
\sigma(n)-2 n=S-p^{k}
$$

and

$$
\begin{aligned}
\left(S-p^{k}\right)^{2} & =(\sigma(n)-2 n)^{2} \\
& =\sigma(n) \\
& =S+p^{k} . \quad(*)
\end{aligned}
$$

If $k \geq 2,(*)$ gives us, by working modulo $p^{2}$, that

$$
S^{2} \equiv S \quad\left(\bmod p^{2}\right) ;
$$

as $S \equiv 1+p\left(\bmod p^{2}\right)$, it follows that

$$
(1+p)^{2} \equiv 1+p \quad\left(\bmod p^{2}\right)
$$


i.e. $p^{2}$ divides $p$, a contradiction. Therefore $k=1, S=1$ and $(*)$ becomes

$$
(1-p)^{2}=1+p,
$$

that is $p^{2}=3 p, p=3$ and $n=p^{k}=3^{1}=3$.

\section{THE MAIN THEOREM}

On the positive side, one has

Proposition 1. If $m$ is a perfect number such that $2 m-1$ is prime, then

$$
n:=m(2 m-1)
$$

is quadratically perfect.

Remark. $m=6(2 m-1=11)$ yields $n=66$, and $m=496(2 m-1=991)$ yields $n=491536$.

Proof. Seeing that $m$ and $2 m-1$ are coprime, one has

$$
\begin{aligned}
\sigma(n)= & \sigma(m(2 m-1)) \\
= & \sigma(m) \sigma(2 m-1) \\
= & 2 m(1+(2 m-1)) \\
& {[\text { as } m \text { is perfect and } 2 m-1 \text { is prime] }} \\
= & 4 m^{2} .
\end{aligned}
$$

Therefore

$$
\begin{aligned}
\sigma(n)-2 n & =4 m^{2}-2 m(2 m-1) \\
& =2 m,
\end{aligned}
$$

and

$$
(\sigma(n)-2 n)^{2}=(2 m)^{2}=4 m^{2}=\sigma(n) ;
$$

$n$ is quadratically perfect.

A partial converse holds :

Theorem 1. Let $n$ be an even quadratically perfect number; then there exists an even perfect number $m$ such that $n=m(2 m-1)$.

Remark. It is well-known (see e.g. [1,Theorem 277], or [2,pp.33-34]) that $m$ can then be written as

$$
m=2^{p-1}\left(2^{p}-1\right)
$$

with $2^{p}-1$ (hence also $p$ ) prime ; $m=6$ corresponds to $p=2$, and $m=496$ to $p=5$. 


\section{The PRoof of Theorem 1}

For convenience, this will be broken up into four lemmas.

Let us write $n=2^{a} x$ ( $a \geq 1, x$ odd $)$; then

$$
\begin{aligned}
\sigma(n) & =\sigma\left(2^{a}\right) \sigma(x) \\
& =\left(2^{a+1}-1\right) \sigma(x) .
\end{aligned}
$$

Lemma 2. There are integers $b, e$ and $f$ such that $\sigma(x)=b e^{2}$ and

$$
b c e^{2}-\left(b c^{2}+1\right) f-e=0 .
$$

Proof. Let us define $b$ as the square-free part of $2^{a+1}-1$; then

$$
2^{a+1}-1=b c^{2}
$$

for some $c \in \mathbf{N}, c \geq 1$. As $\left.2^{a+1}-1 \equiv-1(\bmod 4)\right), 2^{a+1}-1$ is not a square, therefore $b \geq 2$; in fact, $b \geq 3$ as $b$ is odd.

As $(\sigma(n)-2 n)^{2}=\sigma(n)$, we have

$$
\begin{aligned}
b c^{2} \sigma(x) & =\left(2^{a+1}-1\right) \sigma(x) \\
& =\sigma(n) \\
& =(\sigma(n)-2 n)^{2}
\end{aligned}
$$

whence $c$ divides $\sigma(n)-2 n$ :

$$
\sigma(n)-2 n=\epsilon c d,
$$

with $\epsilon \in\{-1,1\}$ and $d \geq 1$. It follows that

$$
b c^{2} \sigma(x)=c^{2} d^{2},
$$

i.e. $b \sigma(x)=d^{2}$, and $b$ divides $d^{2} ; b$ being square-free, $b$ divides $d: d=b e(e \geq 1)$. Therefore $b \sigma(x)=b^{2} e^{2}$, i.e. $\sigma(x)=b e^{2}$. It now appears that

$$
\begin{aligned}
b^{2} c^{2} e^{2}-\left(b c^{2}+1\right) x & =b c^{2} \cdot b e^{2}-2^{a+1} x \\
& =\left(2^{a+1}-1\right) \sigma(x)-2 n \\
& =\sigma(n)-2 n \\
& =\epsilon c d \\
& =\epsilon c b e
\end{aligned}
$$

therefore, $b c$ divides $x$.

Let us then set $x=b c f(f \geq 1)$; as $b>1, b c>1, b e^{2}=\sigma(x) \geq x+f$ and

$$
\begin{aligned}
b^{2} c^{2} e^{2} & =b c^{2} \sigma(x) \\
& \geq b c^{2} x+b c^{2} f \\
& \geq b c^{2} x+b c f \\
& =b c^{2} x+x,
\end{aligned}
$$

thus $\epsilon=1$.

We now get

$$
b^{2} c^{2} e^{2}-\left(b c^{2}+1\right) b c f=c b e,
$$

that is

$$
b c e^{2}-\left(b c^{2}+1\right) f-e=0 .
$$


Lemma 3. $c=1$, i.e. $2^{a+1}-1$ is square-free.

Proof. We know that $b>1$; then

$$
b e^{2}=\sigma(x)=\sigma(b c f) \geq b c f+c f,
$$

and, using Lemma 3.1 :

$$
\begin{aligned}
0 & =b c e^{2}-\left(b c^{2}+1\right) f-e \\
& \geq c(b c f+c f)-\left(b c^{2}+1\right) f-e \\
& =\left(c^{2}-1\right) f-e
\end{aligned}
$$

Assuming that $c>1$, it would follow that $f \leq \frac{e}{c^{2}-1}$, whence that

$$
\begin{aligned}
b c e^{2} & =\left(b c^{2}+1\right) f+e \\
& \leq\left(b c^{2}+1\right) \frac{e}{c^{2}-1}+e \\
& =\frac{e}{c^{2}-1}\left(b c^{2}+c^{2}\right) \\
& =e(b+1) \frac{c^{2}}{c^{2}-1} .
\end{aligned}
$$

It would now appear that

$$
\begin{aligned}
e & \leq \frac{b+1}{b} \frac{c}{c^{2}-1} \\
& \leq \frac{4}{3} \frac{3}{8}=\frac{1}{2}<1
\end{aligned}
$$

a contradiction.

Therefore $c=1$.

Lemma 4. $b$ is prime.

Proof. From Lemmas 3.1 and 3.2 follows that

$$
b e^{2}-e-(b+1) f=0 .
$$

As $x=b c f=b f$, one has $\sigma(b f)=\sigma(x)=b e^{2}$.

We know that $b \geq 3$; let us assume that $b$ is not prime, and let $p$ denote the smallest prime factor of $b$. Then

$$
\sigma(b) \geq b+p+1 \geq b+4
$$

and

whence

$$
f(b+4) \leq f \sigma(b) \leq \sigma(b f)=b e^{2},
$$

and $3 f \leq e$. It follows that

$$
f(b+4) \leq b e^{2}=e+(b+1) f
$$




$$
\begin{aligned}
3 b e^{2} & =3 e+(3 b+3) f \\
& \leq 3 e+(b+1) e \\
& =e(b+4),
\end{aligned}
$$

hence $3 b \leq 3 b e \leq b+4,2 b \leq 4$ and $b \leq 2$, a contradiction.

Therefore $b$ is prime.

Lemma 5. $b$ does not divide $f$.

Proof. Let us assume for a moment that $b$ divides $f$; then, as

$$
b e^{2}=e+b f+f,
$$

$b$ divides $e$. It follows that

$$
b e^{2}=\sigma(b f) \geq b f+f+\frac{f}{b},
$$

whence

$$
\frac{f}{b} \leq b e^{2}-b f-f=e,
$$

thus $f \leq b e$.

Now

$$
\begin{aligned}
b e^{2} & =e+b f+f \\
& \leq e+b^{2} e+b e
\end{aligned}
$$

and

$$
\begin{aligned}
b e & \leq b^{2}+b+1 \\
& =b(b+1)+1 \\
& <b(b+2) .
\end{aligned}
$$

It appears that $e<b+2$, hence $e \leq b+1<2 b$; as $b$ divides $e, e=b$ and

$$
(b+1) f=b e^{2}-e=b^{3}-b,
$$

thus $f=b(b-1)$.

Now we have

$$
\sigma\left(b^{2}(b-1)\right)=\sigma(b f)=b e^{2}=b^{3}=b^{2}(b-1)+b^{2},
$$

an obvious contradiction as

$$
\sigma\left(b^{2}(b-1)\right) \geq b^{2}(b-1)+b^{2}+b+1 .
$$

We conclude that $b$ does not divide $f$. 


\section{Conclusion}

We may now write

$$
\begin{aligned}
(b+1) \sigma(f)= & \sigma(b) \sigma(f) \\
& {[\text { by Lemma 3.3] }} \\
= & \sigma(b f) \\
& {[\text { as } b \text { and } f \text { are coprime, by Lemmas } 3.3 \text { and } 3.4] } \\
= & b e^{2} \\
= & b f+e+f ;
\end{aligned}
$$

thus $b+1$ divides $e: e=(b+1) g$, hence

$$
\begin{aligned}
(b+1) f & =e(b e-1) \\
& =(b+1) g(b(b+1) g-1)
\end{aligned}
$$

and $f=g(b(b+1) g-1)$. In particular, $g$ divides $f$, and $g \neq f$. As

$$
(b+1) \sigma(f)=b f+(b+1) g+f,
$$

we obtain $\sigma(f)=f+g$; therefore $g=1$ and $f$ is prime.

Furthermore, $f=b(b+1)-1$.

Now let $m:=2^{a}\left(2^{a+1}-1\right)=2^{a} b$; then $m$ is perfect $([1$, Theorem 276$]$, or $[2$, p.33] $)$ and even, $2 m-1=2^{a+1} b-1=(b+1) b-1=f$ is prime, and

$$
m(2 m-1)=2^{a} b f=2^{a} x=n .
$$

\section{Final comments}

It is reasonable to expect the converse of Proposition 2.1 to still hold for all odd $m \geq 5$; combined with the proof of the long-standing conjecture that there is no odd perfect number, this would imply the nonexistence of odd quadratically perfect numbers other than 1 and 3 .

I wish to express my gratitude to Dr. Numa Lescot for checking with the help of a computer that there is at least no such number between 5 and $10^{6}$.

\section{REFERENCES}

[1] G. H. Hardy and E. M. Wright An Introduction to the Theory of Numbers, Fifth Edition, Oxford Clarendon Press, 1979.

[2] J. Itard Arithmétique et Théorie des Nombres, PUF, 1963.

[3] E. Lucas Théorie des nombres, Albert Blanchard, Paris, 1958.

\section{AdDress}

Paul LESCOT

Professeur, Université de Rouen

Avenue de l'Université, BP.12

76801 Saint-Étienne-du-Rouvray

FRANCE

e-mail : paul.lescot@univ-rouen.fr 


\section{ACKNOWLEDGEMENT}

I wish to thank the (anonymous) referee, whose comments helped me improve the style and readability of the article. 